PROCEEDINGS OF THE

AMERICAN MATHEMATICAL SOCIETY

Volume 137, Number 5, May 2009, Pages 1801-1805

S 0002-9939(08)09738-4

Article electronically published on November 19, 2008

\title{
ON AN OPEN PROBLEM REGARDING TOTALLY FENCHEL UNSTABLE FUNCTIONS
}

\author{
RADU IOAN BOŢ AND ERNÖ ROBERT CSETNEK
}

(Communicated by N. Tomczak-Jaegermann)

\begin{abstract}
We give an answer to Problem 11.5 posed in Stephen Simons's book From Hahn-Banach to Monotonicity.
\end{abstract}

\section{INTRODUCTION AND PROBLEM FORMULATION}

Before introducing the problem proposed by Stephen Simons, we recall some preliminary notions and results. Throughout this note, $E$ denotes a nontrivial real Banach space, $E^{*}$ its topological dual space and $E^{* *}$ its bidual space. The canonical embedding of $E$ into $E^{* *}$ is defined by $\widehat{~: ~} E \rightarrow E^{* *},\left\langle x^{*}, \widehat{x}\right\rangle:=\left\langle x, x^{*}\right\rangle$, for all $x \in E$ and $x^{*} \in E^{*}$, where $\left\langle x, x^{*}\right\rangle$ denotes the value of the linear continuous functional $x^{*}$ at $x$. For $D \subseteq E$, we denote by $\widehat{D}$ the image of the set $D$ through the canonical embedding, that is, $\widehat{D}=\{\widehat{x}: x \in D\}$.

The indicator function of $D \subseteq E$, denoted by $\delta_{D}$, is defined as $\delta_{D}: E \rightarrow \overline{\mathbb{R}}$,

$$
\delta_{D}(x)= \begin{cases}0, & \text { if } x \in D \\ +\infty, & \text { otherwise }\end{cases}
$$

where $\overline{\mathbb{R}}=\mathbb{R} \cup\{ \pm \infty\}$. For a function $f: E \rightarrow \overline{\mathbb{R}}$ we denote by $\operatorname{dom}(f)=\{x \in$ $E: f(x)<+\infty\}$ its domain and by epi $(f)=\{(x, r) \in E \times \mathbb{R}: f(x) \leq r\}$ its epigraph. We call $f$ proper if $\operatorname{dom}(f) \neq \emptyset$ and $f(x)>-\infty$ for all $x \in E$. The Fenchel-Moreau conjugate of $f$ is the function $f^{*}: E^{*} \rightarrow \overline{\mathbb{R}}$ defined by $f^{*}\left(x^{*}\right)=$ $\sup _{x \in E}\left\{\left\langle x, x^{*}\right\rangle-f(x)\right\}$ for all $x^{*} \in E^{*}$.

Consider $f, g: E \rightarrow \overline{\mathbb{R}}$ to be two arbitrary convex functions. We say that $f$ and $g$ satisfy stable Fenchel duality if for all $x^{*} \in E^{*}$, there exists $z^{*} \in E^{*}$ such that

$$
(f+g)^{*}\left(x^{*}\right)=f^{*}\left(x^{*}-z^{*}\right)+g^{*}\left(z^{*}\right) .
$$

If this property holds for $x^{*}=0$, then $f$ and $g$ satisfy the classical Fenchel duality. The pair $f, g$ is totally Fenchel unstable (see [10]) if $f$ and $g$ satisfy Fenchel duality

Received by the editors December 18, 2007, and, in revised form, July 21, 2008.

2000 Mathematics Subject Classification. Primary 90C25, 90C46; Secondary 42A50, 90C47, $46 \mathrm{~B} 20$.

Key words and phrases. Conjugate function, Fenchel duality, minimax theorem, weak*extreme point.

The first author was partially supported by DFG (German Research Foundation), project WA $922 / 1$.

The second author was supported by a Graduate Fellowship of the Free State Saxony, Germany.

(C)2008 American Mathematical Society Reverts to public domain 28 years from publication 
but

$$
y^{*}, z^{*} \in E^{*} \text { and }(f+g)^{*}\left(y^{*}+z^{*}\right)=f^{*}\left(y^{*}\right)+g^{*}\left(z^{*}\right) \Longrightarrow y^{*}+z^{*}=0 .
$$

We refer the reader to [1] for a geometric characterization of these concepts.

Obviously, stable Fenchel duality implies Fenchel duality, but the converse is not true (see the example in [1, pp. 2798-2799, and Example 11.1 in [10]). Nevertheless, each of these examples (which are given in $\mathbb{R}^{2}$ ) fails when one tries to verify total Fenchel unstability. Surprisingly, in the finite dimensional case, it is still an open question if there exists a pair of functions which is totally Fenchel unstable (see Problem 11.6 in [10]). In the infinite dimensional setting this problem receives an answer, due to the existence of extreme points which are not support points of certain convex sets. Recall that if $C$ is a convex subset of $E$, then $x \in C$ is a support point of $C$ if there exists $x^{*} \in E^{*}, x^{*} \neq 0$ such that $\left\langle x, x^{*}\right\rangle=\sup \left\langle C, x^{*}\right\rangle$. We give below an example, proposed in [10, of a pair $f, g$ which is totally Fenchel unstable.

Example 1.1. Let $C$ be a nonempty, bounded, closed and convex subset of $E$ such that there exists an extreme point $x_{0}$ of $C$ which is not a support point of $C$ (an example of a set $C$ and a point $x_{0}$ with the above-mentioned properties was given in the space $l_{2}$, following an idea due to Jonathan Borwein; see [10]). Take $A:=x_{0}-C, B:=C-x_{0}, f:=\delta_{A}$ and $g:=\delta_{B}$. One can prove that the pair $f, g$ is totally Fenchel unstable (see Example 11.3 in [10]).

Regarding the functions defined in the above example, Stephen Simons asks whether, denoting $E^{*} \backslash\{0\}$ with $\{0\}^{c}$, the following representation of the Minkowski sum of the sets epi $\left(f^{*}\right)$ and epi $\left(g^{*}\right)$ is true:

$$
\operatorname{epi}\left(f^{*}\right)+\operatorname{epi}\left(g^{*}\right)=(\{0\} \times[0, \infty)) \cup\left(\{0\}^{c} \times(0, \infty)\right) .
$$

The justification for this question comes from a similar representation of the set epi $\left(f_{0}^{*}\right)+\operatorname{epi}\left(g_{0}^{*}\right)$, proved in [10] for a pair of functions $f_{0}, g_{0}$ defined on the space $\mathbb{R}^{2}$ in a similar way as in Example 1.1 above (see Example 11.1 and Example 11.2 in 10).

We give in the following a reformulation of this problem (as in [10]). The conjugates of the functions $f$ and $g$ are

$$
\begin{aligned}
& f^{*}\left(y^{*}\right)=\left\langle x_{0}, y^{*}\right\rangle-\inf \left\langle C, y^{*}\right\rangle \geq 0 \text { for all } y^{*} \in E^{*} \text { and } \\
& g^{*}\left(y^{*}\right)=\sup \left\langle C, y^{*}\right\rangle-\left\langle x_{0}, y^{*}\right\rangle \geq 0 \text { for all } y^{*} \in E^{*} .
\end{aligned}
$$

One can use the boundedness of the set $C$ to conclude that $f^{*}$ and $g^{*}$ are continuous functions. The inclusion " $\subseteq$ " in (1.1) holds and, since $(0,0)=(0,0)+(0,0) \in$ $\operatorname{epi}\left(f^{*}\right)+\operatorname{epi}\left(g^{*}\right)$, relation (1.1) is equivalent to

$$
\operatorname{epi}\left(f^{*}\right)+\operatorname{epi}\left(g^{*}\right) \supset E^{*} \times(0, \infty) .
$$

Let us mention that for the implication $(1.2) \Rightarrow(1.1)$, the assumption that $x_{0}$ is not a support point of $C$ is decisive.

In case $E$ is reflexive, this question has a positive answer. Although the proof is given in [10] (Example 11.3), we give the details for the reader's convenience. Let $y^{*} \in E^{*}$ be arbitrary. Consider the functions $h: E^{*} \rightarrow \mathbb{R}$ and $k: E^{*} \rightarrow \mathbb{R}$ defined by $h\left(z^{*}\right):=f^{*}\left(z^{*}\right)$ and $k\left(z^{*}\right):=g^{*}\left(y^{*}-z^{*}\right)$ for all $z^{*} \in E^{*}$. Since $h$ and $k$ are 
continuous, it follows that $h$ and $k$ satisfy Fenchel duality (see Theorem 2.8.7 in [11]). This and the reflexivity of the space $E$ give

$$
-\inf _{E^{*}}[h+k]=(h+k)^{*}(0)=\min _{z \in E}\left[h^{*}(z)+k^{*}(-z)\right] .
$$

A simple computation shows that $h^{*}(z)=f(z)$ and $k^{*}(-z)=g(z)-\left\langle z, y^{*}\right\rangle$, for all $z \in E$. Hence, since $x_{0}$ is an extreme point of $C$,

$$
-\inf _{E^{*}}[h+k]=\min _{E}\left[f+g-y^{*}\right]=\min _{E}\left[\delta_{\{0\}}-y^{*}\right]=0,
$$

so, for all $\varepsilon>0$, there exists $z^{*} \in E^{*}$ such that $h\left(z^{*}\right)+k\left(z^{*}\right) \leq \varepsilon$, that is $f^{*}\left(z^{*}\right)+$ $g^{*}\left(y^{*}-z^{*}\right) \leq \varepsilon$. This means exactly that $\left(y^{*}, \varepsilon\right) \in \operatorname{epi}\left(f^{*}\right)+\operatorname{epi}\left(g^{*}\right)$; hence the proof of (1.2) is complete.

Remark 1.2. Regarding the proof given above, one can easily notice that relation (1.1) is fulfilled if and only if for all $y^{*} \in E^{*}$ and for all $\varepsilon>0$ there exists $z^{*} \in E^{*}$ such that $f^{*}\left(z^{*}\right)+g^{*}\left(y^{*}-z^{*}\right) \leq \varepsilon$. This is equivalent to the statement that there exists $z^{*} \in E^{*}$ such that for all $x, y \in E, f(x)+g(y)-\left\langle x-y, z^{*}\right\rangle \geq\left\langle y, y^{*}\right\rangle-\varepsilon$. Using the Hahn-Banach-Lagrange theorem (see Theorem 1.11 in [10]), this is equivalent to the following: there exists $M \geq 0$ such that for all $x, y \in E, f(x)+g(y)+M \| x-$ $y \| \geq\left\langle y, y^{*}\right\rangle-\varepsilon$; that is to say, there exists $M \geq 0$ such that for all $u, v \in C$, $M\left\|u+v-2 x_{0}\right\| \geq\left\langle v-x_{0}, y^{*}\right\rangle-\varepsilon$.

Following this remark, Stephen Simons proposed the following problem (Problem 11.5 in $[10$ ):

Problem 1.3. Let $C$ be a nonempty, bounded, closed and convex subset of a nonreflexive Banach space $E, x_{0}$ be an extreme point of $C, y^{*} \in E^{*}$ and $\varepsilon>0$. Then does there always exist $M \geq 0$ such that, for all $u, v \in C, M\left\|u+v-2 x_{0}\right\| \geq$ $\left\langle v-x_{0}, y^{*}\right\rangle-\varepsilon$ ? If the answer to this question is positive, then epi $\left(\delta_{x_{0}-C}^{*}\right)+$ $\operatorname{epi}\left(\delta_{C-x_{0}}^{*}\right) \supset E^{*} \times(0, \infty)$.

\section{The solution to Problem 1.3}

We give in this section an answer to Problem 1.3. We show that in the nonreflexive case the answer depends on whether $x_{0}$ is a weak*-extreme point of $C$ or not. We recall that $x_{0}$ is a weak $k^{*}$-extreme point of the nonempty, bounded, closed and convex set $C \subseteq E$ if $\widehat{x_{0}}$ is an extreme point of cl $\widehat{C}$, where the closure is taken with respect to the weak ${ }^{*}$ topology $\omega\left(E^{* *}, E^{*}\right)$ (see $[6]$ ). One can show that if $x_{0}$ is a weak*-extreme point of $C$, then $x_{0}$ is an extreme point of $C$. The history of this notion goes back to the paper of Phelps (see [8]), where the author asked the following: must the image $\widehat{x}$ of an extreme point of $x \in B_{E}$ (the unit ball of $E$ ) be an extreme point of $B_{E^{* *}}$ (the unit ball of the bidual)? We recall that by the Goldstine theorem, the closure of $\widehat{B_{E}}$ in the weak* topology $\omega\left(E^{* *}, E^{*}\right)$ is $B_{E^{* *}}$ (hence the generalization to a nonempty, bounded, closed and convex set is natural). Several papers from the literature deal with this notion; see [2, 3, 4, 6, 7, 8, In the spaces $C(X)$ and $L^{p}(1 \leq p \leq \infty)$ all the extreme points of the corresponding unit balls are weak*-extreme points (see [7]). The first example of a Banach space of the unit ball which contains elements that are not weak ${ }^{*}$-extreme was suggested by K. de Leeuw and proved by Y. Katznelson (see the note added at the end of [8]). If $E$ is a separable Banach space containing an isomorphic copy of $c_{0}$, then $E$ is isomorphic to a strictly convex space $F$ such that $B_{F}$ has no weak*-extreme points (see [7). For the general case when $C$ is a bounded, closed and convex set, we refer to [2] 
and [6] for more on this subject. We recall from [2] the following result: a Banach space $E$ has the Radon-Nikodým property if and only if every bounded, closed and convex subset $C$ of $E$ has a weak*-extreme point. Of course, in a Radon-Nikodým space it is possible that some of the extreme points are not weak ${ }^{*}$-extreme points (see [5] for other equivalent formulations of the Radon-Nikodým property).

Theorem 2.1. We have $E^{*} \times(0, \infty) \subset \operatorname{epi}\left(f^{*}\right)+\operatorname{epi}\left(g^{*}\right)$ if and only if $x_{0}$ is a weak ${ }^{*}$-extreme point of $C$.

Proof. Let $y^{*} \in E^{*}$ and $\varepsilon>0$ be arbitrary. In view of Remark 1.2 the condition $\left(y^{*}, \varepsilon\right) \in \operatorname{epi}\left(f^{*}\right)+\operatorname{epi}\left(g^{*}\right)$ is equivalent to the statement that there exists $z^{*} \in E^{*}$ such that for all $x, y \in E, f(x)+g(y)-\left\langle x-y, z^{*}\right\rangle \geq\left\langle y, y^{*}\right\rangle-\varepsilon$, which is nothing else than there exists $z^{*} \in E^{*}$ such that for all $u, v \in C,\left\langle u+v-2 x_{0}, z^{*}\right\rangle+\left\langle x_{0}-v, y^{*}\right\rangle \geq$ $-\varepsilon$. Hence the inclusion $E^{*} \times(0, \infty) \subset \operatorname{epi}\left(f^{*}\right)+\operatorname{epi}\left(g^{*}\right)$ is fulfilled if and only if

$$
\inf _{y^{*} \in E^{*}} \sup _{z^{*} \in E^{*}} \inf _{(u, v) \in C \times C}\left[\left\langle u+v-2 x_{0}, z^{*}\right\rangle+\left\langle x_{0}-v, y^{*}\right\rangle\right] \geq 0 .
$$

Take $y^{*} \in E^{*}$. For $z^{*} \in E^{*}$, we have

$$
\begin{aligned}
& \quad \inf _{(u, v) \in C \times C}\left[\left\langle u+v-2 x_{0}, z^{*}\right\rangle+\left\langle x_{0}-v, y^{*}\right\rangle\right] \\
& =\inf _{(u, v) \in \widehat{C} \times \widehat{C}}\left[\left\langle z^{*}, u+v-2 \widehat{x_{0}}\right\rangle+\left\langle y^{*}, \widehat{x_{0}}-v\right\rangle\right] \\
& =\inf _{(u, v) \in \operatorname{cl} \widehat{C} \times \operatorname{cl} \widehat{C}}\left[\left\langle z^{*}, u+v-2 \widehat{x_{0}}\right\rangle+\left\langle y^{*}, \widehat{x_{0}}-v\right\rangle\right],
\end{aligned}
$$

where the first equality follows by the definition of the canonical embedding and the second one is a consequence of the continuity (in the weak ${ }^{*} \operatorname{topology} \omega\left(E^{* *}, E^{*}\right)$ ) of the functions $\left\langle x^{*}, \cdot\right\rangle: E^{* *} \rightarrow \mathbb{R}$, for all $x^{*} \in E^{*}$. The set $C$ being bounded, we use the celebrated Banach-Alaoglu theorem to conclude that the set $\operatorname{cl} \widehat{C}$ is weak*compact. We apply a minimax theorem (see for example Theorem 3.1 in [9]) and obtain that

$$
\begin{aligned}
& \sup _{z^{*} \in E^{*}} \inf _{(u, v) \in C \times C}\left[\left\langle u+v-2 x_{0}, z^{*}\right\rangle+\left\langle x_{0}-v, y^{*}\right\rangle\right] \\
& =\sup _{z^{*} \in E^{*}} \inf _{(u, v) \in \operatorname{cl} \widehat{C} \times \operatorname{cl} \widehat{C}}\left[\left\langle z^{*}, u+v-2 \widehat{x_{0}}\right\rangle+\left\langle y^{*}, \widehat{x_{0}}-v\right\rangle\right]
\end{aligned}
$$

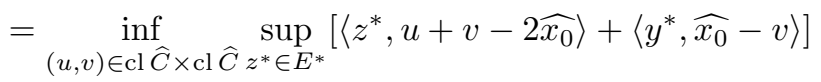

$$
\begin{aligned}
& =\inf _{\substack{(u, v) \in \operatorname{cl} \widehat{C} \times \operatorname{cl} \widehat{C} \\
u+v=2 \widehat{x_{0}}}}\left\langle y^{*}, \widehat{x_{0}}-v\right\rangle .
\end{aligned}
$$

Thus

$$
\begin{aligned}
& \inf _{y^{*} \in E^{*}} \sup _{z^{*} \in E^{*}} \inf _{(u, v) \in C \times C}\left[\left\langle u+v-2 x_{0}, z^{*}\right\rangle+\left\langle x_{0}-v, y^{*}\right\rangle\right] \\
& =\inf _{y^{*} \in E^{*}} \inf _{\substack{(u, v) \in \operatorname{cl} \widehat{C} \times \operatorname{cl} \widehat{C} \\
u+v=2 \widehat{x_{0}}}}\left\langle y^{*}, \widehat{x_{0}}-v\right\rangle=\inf _{\substack{u, v) \in \operatorname{cl} \widehat{C} \times \operatorname{cl} \widehat{C} \\
u+v=2 \widehat{x_{0}}}} \inf _{y^{*} \in E^{*}}\left\langle y^{*}, \widehat{x_{0}}-v\right\rangle \\
& =\inf _{\substack{(u, v) \in \operatorname{cl} \widehat{C} \times \operatorname{cl} \widehat{C} \\
u+v=2 \widehat{x_{0}}}}-\delta_{\left\{\widehat{x_{0}}\right\}}(v) .
\end{aligned}
$$

Since this has the value 0 if $x_{0}$ is a weak*-extreme point of $C$, and the value $-\infty$ otherwise, this completes the proof of (2.1). 
Remark 2.2. The above result gives the solution to Problem 1.3 (see Remark 1.2); namely, the answer is positive if and only if $x_{0}$ is a weak ${ }^{*}$-extreme point of $C$. Let us mention that the closedness of the set $C$, requested in [10, is not needed anymore for this result.

\section{ACKNOWLEDGEMENTS}

The authors would like to express their gratitude to Stephen Simons for carefully reading a preliminary version of the paper and for providing an alternative proof to Theorem 1 based on Fenchel duality. The authors are thankful also to an

anonymous referee for remarks and suggestions which improved the quality of the paper.

\section{REFERENCES}

1. R. I. Bot, G. Wanka, A weaker regularity condition for subdifferential calculus and Fenchel duality in infinite dimensional spaces, Nonlinear Anal. 64 (12) (2006), 2787-2804. MR.2218547 (2006k:49038)

2. J. Bourgain, A geometric characterization of the Radon-Nikodým property in Banach spaces, Compos. Math. 36 (1) (1978), 3-6. MR515034 (80h:46018)

3. S. Dutta, T. S. S. R. K. Rao, On weak*-extreme points in Banach spaces, J. Convex Anal. 10 (2) (2003), 531-539. MR2044435 (2004m:46025)

4. B. V. Godun, B.-L. Lin, S. L. Troyanski, On the strongly extreme points of convex bodies in separable Banach spaces, Proc. Amer. Math. Soc. 114 (3) (1992), 673-675. MR1070518 (92f:46014)

5. R. E. Huff, P. D. Morris, Dual spaces with the Krein-Milman property have the RadonNikodým property, Proc. Amer. Math. Soc. 49 (1) (1975), 104-108. MR0361775 (50:14220)

6. K. Kunen, H. Rosenthal, Martingale proofs of some geometrical results in Banach space theory, Pacific J. Math. 100 (1) (1982), 153-175. MR661446 (83k:46023)

7. P. Morris, Disappearance of extreme points, Proc. Amer. Math. Soc. 88 (2) (1983), 244-246. MR695251 (85b:46021)

8. R. R. Phelps, Extreme points of polar convex sets, Proc. Amer. Math. Soc. 12 (2) (1961), 291-296. MR0121634 (22:12368)

9. S. Simons, Minimax and Monotonicity, Lecture Notes in Math., 1693, Springer-Verlag, Berlin, 1998. MR $1723737(2001 \mathrm{~h}: 49002)$

10. S. Simons, From Hahn-Banach to Monotonicity, second edition, Lecture Notes in Math., 1693, Springer, New York, 2008. MR2386931

11. C. Zălinescu, Convex Analysis in General Vector Spaces, World Scientific, River Edge, NJ, 2002. MR $1921556(2003 \mathrm{k}: 49003)$

Faculty of Mathematics, Chemnitz University of Technology, D-09107 Chemnitz, GERMAnY

E-mail address: radu.bot@mathematik.tu-chemnitz.de

Faculty of Mathematics, Chemnitz University of Technology, D-09107 Chemnitz, GERMANY

E-mail address: robert.csetnek@mathematik.tu-chemnitz.de 